\title{
La formación docente en línea: experiencias con MOOCs en Sonora (México)
}

\author{
Online In-Service Teacher Training: Experiences With MOOCs in Sonora (Mexico)
}

La formació docent en línia: experiències amb MOOCs en Sonora (Mèxic)

\author{
María Lorena Ballesteros-lbarra ${ }^{1}$, Martín Alonso Mercado-Varela ${ }^{1, *}$, Nancy \\ Janett García-Vázquez ${ }^{2}$ \\ 1 | Dpto. de Educación, Instituto Tecnológico de Sonora, Sonora (México) \\ 2 | Escuela de Humanidades y Educación, Tecnológico de Monterrey, Nuevo León (México) \\ *Autor para correspondencia: martin.mercado@itson.edu.mx (Martín Alonso Mercado-Varela)
}

Recibido: 10/10/2019 | Aceptado: 09/12/209 | Publicado: 20/12/2019

\section{Cómo citar:}

Ballesteros-Ibarra, M.L., Mercado-Varela, M.A. y García-Vázquez, N.J. (2019). La formación docente en línea: experiencias con MOOCs en Sonora (México). Research in Education and Learning Innovation Archives, 23,62-79. 10.7203/realia.23.15903

Copyright: El/La Autor/a. Open Access: Este es un artículo de acceso abierto distribuido bajo los términos de la licencia Creative Commons AttributionNonCommercial-ShareAlike 4.0 International (CC BY-NC-SA 4.0)

Financiación: Esta investigación es producto del proyecto PROFAPI_2019_0140 'Formación continua de docentes a través de ambientes de aprendizaje masivo: el caso de Sonora', financiado por el Programa de Fomento y Apoyo a la Investigación (PROFAPI 2019).
RESUMEN: El propósito de este estudio fue conocer las perspectivas que un grupo de profesores del estado de Sonora (México) tienen sobre la formación en línea y sobre los cursos en línea masivos y abiertos (MOOCs, por sus siglas en inglés) “Colección de Aprendizajes Clave”. Estos MOOCs fueron diseñados para dar a conocer a los profesores de educación básica los planteamientos del nuevo modelo educativo derivado de la reforma de 2013. El enfoque aplicado en la investigación fue el cualitativo; los datos se colectaron por medio de entrevistas semiestructuradas a seis profesoras de primaria. Las perspectivas de las profesoras en cuanto a la formación en línea se concentraron en las categorías de análisis: formación continua en línea versus presencial, requerimientos de participación de la formación continua en línea, y retos de la formación continua en línea de docentes; y en cuanto a los MOOCs, se categorizaron en: dimensión didáctico-pedagógica de los cursos, masividad y ausencia de tutor, infraestructura tecnológica implementada, y el papel de la autoridad escolar para su implementación. Los hallazgos evidencian una postura abierta de las profesoras hacia la formación en línea; no obstante, su disposición a participar en ella se ve influida por sus experiencias previas en esta modalidad. Asimismo, los hallazgos ponen de manifiesto la necesidad de promover un compromiso conjunto, tanto de autoridades educativas como de maestros, para implementar exitosamente estrategias masivas de formación docente como es el caso de los MOOCs.

PALABRAS CLAVES: formación de docentes; educación a distancia; MOOCs

ABSTRACT: The purpose of this study was to know the perspectives that a group of teachers from the state of Sonora (Mexico) have on online training and on the massive open online courses (MOOCs) "Key Learning Collection". These MOOCs were designed to make basic education teachers aware of the approaches of the new educational model derived from the 2013 reform. The approach applied in the research was the qualitative one; the data was collected through semi-structured interviews with six primary school teachers. Teachers' perspectives regarding online training focused on the categories of analysis: online versus face-to-face in-service teacher training, participation requirements of online in-service teacher training, and challenges of online in-service teacher training; and regarding MOOCs, they were categorized into: didactic-pedagogical dimension of the courses, massiveness and absence of tutor, implemented technological infrastructure, and the role of the school authority for its implementation. The findings show an open position of teachers towards online training; however, their willingness to participate in it is influenced by their previous experiences in this modality. Likewise, findings highlight the need to promote a joint commitment, both of educational authorities and teachers, to successfully implement massive teacher training strategies 
such as MOOCs.

KEYWORDS: Teacher Training; Distance Education; MOOCs

RESUM: El propòsit d'aquest estudi va ser conèixer les perspectives que un grup de professors de l'estat de Sonora (Mèxic) tenen sobre la formació en línia i sobre els cursos en línia massius i oberts (MOOCs, per les seues sigles en anglés) "Col-lecció d'Aprenentatges Clau". Aquests MOOCs van ser dissenyats per a donar a conèixer als professors d'educació bàsica els plantejaments del nou model educatiu derivat de la reforma de 2013. L'enfocament aplicat en la investigació va ser el qualitatiu; les dades es van col-lectar per mitjà d'entrevistes semiestructurades a sis professores de primària. Les perspectives de les professores quant a la formació en línia es van concentrar en les categories d'anàlisis: formació contínua en línia versus presencial, requeriments de participació de la formació contínua en línia, i reptes de la formació contínua en línia de docents; i quant als MOOCs, es van categoritzar en: dimensió didàctic-pedagògica dels cursos, masivitat i absència de tutor, infraestructura tecnològica implementada, i el paper de l'autoritat escolar per a la seua implementació. Les troballes evidencien una postura oberta de les professores cap a la formació en línia; no obstant això, la seua disposició a participar en ella es veu influïda per les seues experiències prèvies en aquesta modalitat. Així mateix, les troballes posen de manifest la necessitat de promoure un compromís conjunt, tant d'autoritats educatives com de mestres, per a implementar reeixidament estratègies massives de formació docent com és el cas dels MOOCs.

PARAULES CLAU: formació de docents; educació a distància; MOOCs

\section{Notas de aplicación práctica}

\section{Qué se sabe sobre este tema}

- Los MOOCs representan una nueva estrategia de formación en línea para profesores. En México, la implementación de esta estrategia en la formación continua de profesores de educación básica pública es reciente, por lo que el incremento de su estudio científico aún es necesario.

\section{Qué aporta este trabajo}

- Presenta y analiza las perspectivas de profesoras de primaria sobre la implementación de MOOCs como estrategia de formación continua en línea.

\section{Implicaciones para la práctica y/o política}

- Se recomienda la promoción de un compromiso conjunto entre autoridades educativas, diseñadores instruccionales, expertos en contenido y facilitadores, que permita potenciar las ventajas que representan los cursos en línea masivos y abiertos para la formación permanente de los profesores en servicio.

\section{INTRODUCCIÓN}

La educación en línea ha resignificado la forma de compartir conocimiento. El acceso a internet y los nuevos dispositivos electrónicos han hecho posible que la educación desarrolle nuevas formas de gestión de los procesos de enseñanza y aprendizaje (Avello-Martínez y Duart, 2016; Patru y Balaji, 2016). Esto ha permitido que las oportunidades para aprender se multipliquen, y existan entornos donde el tiempo y el espacio son elementos adaptables a las características y necesidades personales.

Actualmente, los docentes no sólo se enfrentan a la tarea de enseñar con las Tecnologías de la Información y Comunicación (TIC) sino también a la de aprender a través de ellas en su proceso de formación continua. De acuerdo con Lugo, Lion, y Vaillant (2018) cada vez más profesores buscan oportunidades de desarrollo profesional en la web, ya que ahí pueden encontrar una amplia variedad de opciones flexibles, versátiles y de bajo costo.

En sus resultados más recientes, el Estudio Internacional sobre Enseñanza y Aprendizaje (TALIS, por sus siglas en inglés) reportó que el 54\% de los profesores de los 
países participantes restringía su participación en actividades de desarrollo profesional docente por incompatibilidad con su horario laboral. Las siguientes dos barreras más reportadas fueron falta de incentivos para comprometerse en estas actividades y los costos de participación (OCDE, 2019) . La formación continua, a través de la modalidad en línea, representa una opción con grandes posibilidades para solventar parte de estas problemáticas que experimentan actualmente los profesores.

La UNESCO (2015) reconoce que la tecnología es un apoyo valioso en la formación docente, por lo que propone hacer uso intensivo de soluciones basadas en ella. Una diversidad de estudios se ha hecho bajo esta propuesta, entre ellos los de CaberoAlmenara, Gallego-Pérez, Puentes, y Jiménez (2018); Kannan y Narayanan (2015); Navareño y Rincón (2017); Samperio y Barragán (2018), que exploran la modalidad en línea como una vía para la formación de profesores en servicio.

Una tendencia actual de formación continua en línea son los Cursos en Línea, Masivos y Abiertos (MOOC, por sus siglas en inglés). Algunos autores han sostenido que los MOOCs pueden complementar eficazmente los métodos tradicionales de desarrollo profesional de los docentes debido a su alcance masivo, su facilidad de acceso y porque plantean grandes oportunidades para la creación de comunidades de aprendizaje (Chofré y Calvet, 2014; Ji y Cao, 2016; Jobe, Stlund, y Svensson, 2014; Palmer, 2015; Salmon, Gregory, Lokuge, y Ross, 2015). Al respecto, Misra (2018) agrega que no sólo es preciso conocer las promesas de estas tecnologías sino también sus desafíos e implicaciones. Las mismas características de masividad y apertura representan retos en la formación, y al estar en aumento el uso de esta alternativa educativa, es preciso analizar desde diferentes enfoques las iniciativas de esta modalidad.

En México se ha comenzado a explorar el uso de MOOCs como vía de formación docente a gran escala (Alemán, Sancho-Vinuesa, y Gómez, 2015; García, Ibarra, y Sandoval, 2018; Ramírez y Casillas, 2018; Rivera-Vázquez y Ramírez-Montoya, 2015). Recientemente, la Secretaría de Educación Pública (SEP) implementó un programa de formación a docentes de educación básica, a través de MOOCs, para la implementación de los programas de estudio derivados de la reforma educativa de 2013. A partir de este programa, en enero de 2018 dieron inicio los cursos masivos para formar a más de un millón de profesores de alrededor de 226,000 escuelas de nivel básico en el país (Vicenteño, 2018). Esta iniciativa tuvo por nombre "Colección de Aprendizajes Clave".

El presente estudio se enmarca en esta iniciativa nacional de formación continua en línea, a través de MOOCs, para profesores de educación primaria en México. Desde un enfoque de investigación cualitativo, se tuvo el propósito de conocer las perspectivas que los profesores tienen sobre la educación en línea, y en cursos masivos, con base en sus experiencias formativas con este enfoque. Se entrevistó a un grupo de maestras de primaria y a partir de la información recuperada se discuten los aspectos que delinean su participación en este tipo de cursos, así como algunos retos que indican como parte de esta modalidad de formación continua.

\section{MARCO TEÓRICO DE REFERENCIA}

\subsection{Formación continua en línea de docentes}

Actualmente las TIC han cambiado procesos importantes para el desarrollo social, como la forma en que se accede, produce, difunde y consume la información y el conocimiento. Esto ha impactado muchas áreas de la vida humana, incluyendo la educación (Colomer-Rubio, Sáiz-Serrano, y Bel-Martínez, 2018; Marín Trejo, 2017). De acuerdo con Gárate-Carrillo y Cordero-Arroyo (2019) uno de los procesos educativos que ha visto este impacto es la formación continua del profesorado, que ha aprovechado nuevas modalidades de entrega como la modalidad en línea. 
La formación continua es entendida por Imbernón (2001) como un ejercicio reflexivo que los profesores hacen a lo largo de toda su carrera profesional con el objetivo de innovar su función. La formación permanente de los profesores en México es un proceso transversal, que se caracteriza por tener dos vertientes: (1) la individual, que es el trayecto que sigue el docente para lograr su ingreso, promoción y reconocimiento, y (2) la colectiva, a través de la cual se atiende a la escuela o colegiado escolar (CorderoArroyo, 2015). Para lograr los propósitos de formación continua, se ofertan una variedad de estrategias y recursos atendiendo aspectos específicos de los conocimientos, habilidades o actitudes que los docentes requieren reforzar. Entre los dispositivos más comunes empleados para la formación continua de docentes se enlistan los cursos, talleres, conferencias, coloquios, mesas redondas y seminarios (Ávalos, 2007; Davini, 2015). En el nuevo reporte de TALIS (OCDE, 2019) se agregaron también los cursos o seminarios en línea como actividades formativas para los docentes, mismos que no habían sido explicitados en los dos reportes anteriores de 2008 y 2013, lo cual advierte la atención dirigida a esta modalidad.

La formación continua en línea de profesores es una modalidad en la que: (a) los programas se diseñan con recursos tecnológicos y se soportan por la web; (b) se brindan experiencias interactivas, ya que los recursos combinan texto, video y sonido; y (c) la comunicación es generalmente asincrónica ya que los participantes no necesitan estar conectados a la red al mismo tiempo (NRC, 2007). Entre las ventajas que se le adjudican a la modalidad de entrega en línea, están su flexibilidad pues se adapta a los diversos horarios de los profesores (Dede, 2006), su potencial de crear redes de aprendizaje (Davini, 2015; NRC, 2007) y la posibilidad de generar conocimiento por medio de la discusión constante (Dede, Ketelhut, Whitehouse, Breit, y McCloskey, 2009). Por su parte, algunas desventajas identificadas que también es preciso reconocer, son la falta de conocimientos y experiencia de los profesores respecto a la modalidad en línea, lo que dificulta que se acerquen a esta opción de formación, el poco acceso que algunos profesores tienen a la tecnología, y la falta de apoyo administrativo y financiero que también algunos docentes experimentan (NRC, 2007).

\subsection{Los MOOCs en la formación continua en línea de docentes}

Para Patru y Balaji (2016) hablar de MOOC es entrar a un territorio de innovación y experimentación; aclaran que el concepto aún está abierto a la interpretación, pero consideran que la mayoría de las definiciones coinciden en cuatro características básicas:

- Cursos: los MOOCs son, en primer lugar, cursos, por tanto, esta condición refiere a que ofrecen una experiencia completa de aprendizaje, es decir, tienen objetivos de aprendizaje, están organizados a través de una estructura curricular específica al mismo tiempo que proporcionan materiales y herramientas de evaluación (Patru y Balaji, 2016). Castaño y Cabero (2013) sostienen que deberían tener fechas de inicio y cierre; sin embargo, Jansen, Schuwer, Teixeira, y Aydin (2015) argumentan que este es un tema en debate y que la decisión de tener fechas es de la institución.

- Masividad: lo masivo hace referencia a que fue pensado, diseñado e implementado para atender a un gran número de participantes (Chiappe-Laverde, Hine, y Martínez-Silva, 2015).

- En línea: los MOOCs se distribuyen a través de internet, por lo que cualquier persona con un dispositivo con este servicio puede tener acceso a ellos.

- Abierto: el carácter abierto de los MOOCs puede ser entendido de dos formas: (1) no tienen requisitos de ingreso; y (2) son gratuitos. Marauri (2014) destaca que la apertura es relativa puesto que en realidad las instituciones son las encargadas de 
fijar los requisitos; y Selwyn, Bulfin, y Pangrazio (2015) aclaran que si bien los hay gratuitos, puede ser que no todos lo sean completamente.

Sobre su uso como herramienta de formación docente, algunas investigaciones han concluido que los MOOCs son una opción viable ya que facilitan el acceso a la formación permanente y minimizan las barreras geográficas y temporales (Gonçalves, Chumbo, Torres, y Gonçalves, 2016; Karlsson, Godhe, Bradley, y Lindström, 2014; Koukis y Jimoyiannis, 2019; Koutsodimou y Jimoyiannis, 2015; Laurillard, 2016). Además, Wang, Chen, Fan, y Zhang (2018) sostienen que también son una vía de formación que posibilita la equidad, ya que permiten que los docentes de regiones desfavorecidas puedan acceder a cursos de instituciones universitarias de elevada calidad.

Aunado a las diversas experiencias con resultados favorables en la formación de profesores, también se han reportado algunos retos o desafíos, como el carácter impersonal de las interacciones derivado de la misma masividad de participantes (Salmon et al., 2015), y las bajas tasas de finalización (Benet, Sanahuja, García, y Nieto, 2018). Por su parte, Langseth y Haugsbakken (2016) analizan el hecho de que algunos docentes no tienen experiencia en el uso de la tecnología como una herramienta de aprendizaje formal. Tanto las ventajas, como sus desafíos, animan a explorar con más detalle la modalidad en línea como una oportunidad dentro de la formación continua de los maestros.

\section{MÉTODO}

Esta investigación se realizó bajo el enfoque metodológico cualitativo. Las interrogantes que motivaron la presente investigación fueron: ¿Cuáles son las perspectivas de un grupo de profesores de educación primaria de Ciudad Obregón, Sonora, respecto a la formación continua en línea? ¿Cuáles son las experiencias de un grupo de profesores de educación primaria de Ciudad Obregón, Sonora, derivadas de su participación en los MOOCs "Colección de Aprendizajes Clave"? A continuación, se describe el contexto de la investigación, así como la estrategia de selección de los participantes, y los procesos de recolección y análisis de datos.

\subsection{Contexto}

En México, la reforma educativa de 2013 dio paso a la revisión de la organización que había tenido el trayecto curricular de la educación básica. Como resultado, la autoridad educativa federal propuso un nuevo Modelo Educativo que contempló una reestructuración de los componentes del sistema educativo. Por lo que, en 2017 se publicó en el Diario Oficial de la Federación el Acuerdo 12/10/17 en el que se establecieron el Plan y los Programas de estudio para la Educación Básica: "Aprendizajes Clave para la Educación Integral”. Este documento fundamentalmente señala la importancia de asegurar una educación de calidad y el máximo logro de aprendizajes para niñas, niños y jóvenes mexicanos (DOF, 2017).

Uno de los pasos posteriores a esta acción, se relacionó con la manera de abordar la formación del personal docente para la implementación de los nuevos planteamientos en las aulas. Ante la necesidad que se enfrentaba, la Estrategia Nacional de Formación Continua 2018 incluyó en la tercera línea de formación, referida a los temas prioritarios del Modelo Educativo, la oferta del programa "Colección de Aprendizajes Clave" consistente en 19 cursos en formato MOOC. De estos cursos, uno fue dirigido a educación inicial, uno a prescolar, seis a primaria, ocho a secundaria, uno a personal directivo y uno para cada asignatura transversal: inglés y educación física. Este programa de formación continua en línea, propuso acercar a los docentes de educación básica al conocimiento de los planteamientos de los nuevos programas de estudio y propiciar su aplicación con los alumnos (SEP, 2018) (ver Figura 1). 


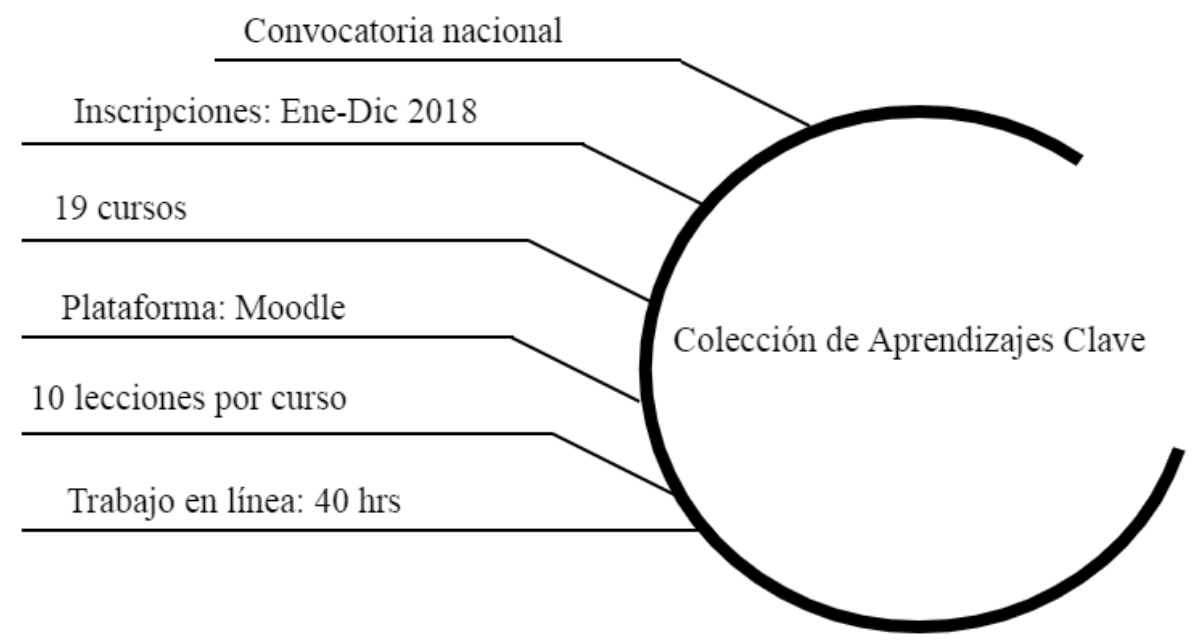

Figura 1. Colección de Aprendizajes Clave

Sonora es un estado de la región noroeste de México. Este estado definió en 2018 su Estrategia Estatal de Formación Continua para docentes de educación básica, la cual fue un esfuerzo entre la Secretaría de Educación Pública, a través de sus programas y apoyos federales, y el propio estado de Sonora, con el propósito de atender las diferentes necesidades de los profesores. En concordancia con el Plan Estatal de Desarrollo 2016-2021, en su reto cuatro, el estado de Sonora se propuso acciones que permitieran "elevar la calidad de la educación para impulsar la creatividad, el ingenio, las competencias y los valores fundamentales de los sonorenses, potencializando el talento del personal docente y desarrollando sus capacidades de aprendizaje" (DGFC, 2018, p.4). Más específicamente, en la estrategia 4.3 del Plan de Desarrollo "Establecer para Sonora un programa sistemático de formación y profesionalización docente", en su línea 5.3.1. "Reivindicar la función del maestro (a) como el más valioso apoyo a los procesos de transformación educativa, otorgándole el reconocimiento y opciones de actualización, capacitación y superación profesional", se soportó el compromiso del estado de Sonora con la formación continua de sus profesores (DGFC, 2018, p. 4).

En concordancia con la Estrategia Nacional de Formación Continua, el estado de Sonora especificó la necesidad de atender la oferta propuesta por la Dirección General de Formación Continua (DGFC), consistente en la implementación de una serie de cursos denominados "Colección de Aprendizajes Clave" (DGFC, 2018, p.14). En seguimiento a esto, los profesores de educación básica de Sonora fueron partícipes de dicha iniciativa de formación. Concretamente este estudio se circunscribió al contexto de Ciudad Obregón, Sonora, por ser la zona a la cual tenían acceso inmediato los investigadores.

\subsection{Participantes}

Se consideraron como participantes del estudio a aquellos que fueran informantes clave, que permitieran obtener información para responder a las preguntas de investigación, y que los investigadores pudieran tener contacto directo con ellos. Los siguientes fueron los criterios de selección: (1) docentes en servicio de primaria pública; y (2) con la experiencia de participación en actividades de formación continua bajo la modalidad en línea, preferentemente en los MOOCs "Colección de Aprendizajes Clave". Dado que no se tenían bases de datos que permitieran seleccionar participantes con estos criterios, se utilizó la estrategia de muestreo de bola de nieve, contactando a los participantes por recomendación. En total se contó con la participación de seis profesoras de primaria egresadas de la Escuela Normal (ver Tabla 1). 
Tabla 1. Características de las participantes

\begin{tabular}{lccc}
\hline Código del participante & Edad & Grado que atiende & $\begin{array}{c}\text { Participación en la 'Colección } \\
\text { de Aprendizajes Clave' }\end{array}$ \\
\hline P1 & 26 & $1^{\circ}$ & Sí \\
P2 & 34 & $4^{\circ}$ & No \\
P3 & 47 & $5^{\circ}$ & Sí \\
P4 & 26 & $3^{\circ}$ & Sí \\
P5 & 40 & $4^{\circ}$ & Sí \\
P6 & 45 & $6^{\circ}$ & Sí \\
\hline
\end{tabular}

\subsection{Recolección y análisis de datos}

La técnica de recolección de información fue la entrevista semiestructurada, que ofrece la posibilidad de hacer preguntas adicionales cuando es necesario profundizar o clarificar algunos temas (Sandín, 2003). En esta fase, se atendieron especialmente los aspectos éticos como el consentimiento informado, contar con la autorización de los participantes para ser grabados en audio y ofrecerles la garantía de confidencialidad y anonimato, con el compromiso de hacer uso de la información exclusivamente para los fines de la investigación (Álvarez Gayou, 2003; Creswell, 2007).

Para el procesamiento de los datos las entrevistas fueron grabadas y posteriormente fielmente transcritas. El total de tiempo de las grabaciones fue de 02 horas 06 minutos 40 segundos. Fue un proceso iterativo que implicó la construcción de categorías de análisis desde los datos recolectados. Se utilizó el software MAXQDA para la gestión, depuración y análisis de datos. Siguiendo las directrices de Forman y Damschroder (2008) el análisis de datos cualitativo se realizó en tres momentos: (1) inmersión; (2) reducción; e (3) interpretación de los datos.

La inmersión tuvo por objetivo familiarizarse con los datos; esto consistió en leer reiteradamente las transcripciones de cada una de las entrevistas y simultáneamente escribir memos con reflexiones iniciales sobre los datos. Una vez que se tuvo un sentido global de esta información, se procedió al segundo momento, el de reducción; en esta etapa se codificaron los segmentos de texto relevantes para responder a la pregunta de investigación y posteriormente estos códigos se agruparon en categorías. Finalmente, con base en estas categorías se describieron e interpretaron los datos para responder a la pregunta de investigación.

\section{RESULTADOS}

Por medio de las entrevistas, se condujo a los participantes a través de distintos tópicos que permitieran conocer sus perspectivas respecto a la modalidad de formación en línea, así como las experiencias derivadas de su participación en la iniciativa de formación "Colección de Aprendizajes Clave". Esta información se describe a continuación en cuatro categorías que emergieron del análisis cualitativo de los datos (ver Figura 2). En las primeras tres categorías se englobaron las perspectivas que los docentes manifestaron sobre la formación continua en línea, y en la cuarta categoría se incluye la información relacionada concretamente con su experiencia en los MOOCs "Colección de Aprendizajes Clave".

\subsection{Formación continua en línea versus presencial}

El primer aspecto que se indagó es qué entienden las profesoras por educación en línea; la definieron como una nueva modalidad de aprendizaje que se basa en un entorno digital, asocian a ella la idea de tener una comunicación asíncrona con un asesor donde 


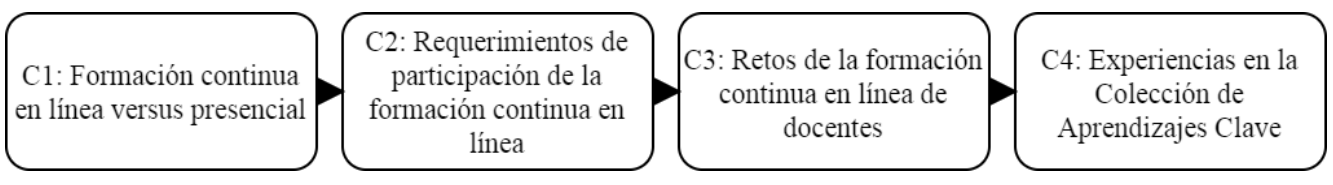

Figura 2. Categorías de análisis

el intercambio de recursos se da por medio de internet. Su percepción la compartieron en función de las diferencias que observan entre los esquemas presencial y virtual; estos hallazgos se concentraron en dos sentidos: (1) ventajas y (2) desventajas. En cuanto a las primeras, mencionaron cuatro ventajas principales de la educación en línea frente a la presencial: (a) flexibilidad de tiempo; (b) flexibilidad de espacio; (c) reducción de costos y (d) formación directa en lugar de "en cascada". En cuanto a las desventajas se resaltaron principalmente tres: (a) retroalimentación no inmediata de un tutor; (b) falta de claridad en las actividades; y (c) ausencia de interacción cara a cara.

Sobre la ventaja de flexibilidad de tiempo, una de las participantes mencionó: “...en línea se ajusta a tu tiempo, sabes que tienes dos semanas o un mes para realizarlo, entonces yo voy a saber de tal manera qué días voy a trabajar o a qué hora se me presta para realizar mi trabajo..." (P1). Respecto a la ventaja de flexibilidad de espacios, las docentes subrayan que la formación presencial les exige estar en un lugar, a una hora estipulada y tienen que ajustarse a ello. Por el contrario, explican que un proceso en línea les da la libertad de estudiar desde cualquier punto con acceso a internet, incluso desde su casa. Esto les proporciona otra ventaja, que es la reducción de gastos al no tener que trasladarse a un lugar específico; igualmente el factor económico se ve favorecido, al encontrar diversas opciones de formación en la red a bajo costo. Así lo perciben, al indicar concretamente: "nos podemos encontrar en línea muchos cursos o talleres que son totalmente gratis, entonces ahí no estamos invirtiendo en eso y el hecho de ir a un lugar y trasladarte pues también genera un costo" (P4).

Finalmente, las docentes identifican una cuarta ventaja asociada con la idea de que en la formación en línea no hay intermediarios entre la información o conocimiento y la persona que aprende, por ello, creen que la interpretación que logran es más precisa en contraste con la denominada "formación en cascada". Este tipo de formación es el formato usual a través del cual los profesores reciben actualizaciones respectivas a su profesión; y consiste en que la información desciende de forma vertical, a través de los distintos niveles de competencia de la autoridad educativa, hasta llegar a los docentes. Una de las participantes expresó esta ventaja de la siguiente manera:

Principalmente considero que cuando es formación en cascada, ahí la información se va distorsionando, ya no llega completamente como debe ser [...] cuando ya me enfrenté al curso como tal, ya aprendí verdaderamente de lo que se trataba, entonces estaba la información tal cual, no era la interpretación de alguien más (P4).

En relación con las desventajas, la mayoría de las participantes señalaron cuestiones que tienen que ver con la ausencia física de un tutor; manifiestan que la retroalimentación no puede ser inmediata, si hay alguna duda tienen que recurrir a contacto por vía electrónica y muchas veces la respuesta tarda. Así lo expresa una de las entrevistadas: "si estoy en una computadora y tengo dudas pues sí le puedo mandar al maestro: 'maestro, ¿me podría explicar este tema?' pero me responde ¿qué?, ¿una?, ¿tres?... una semana después" (P2). A esto se le suma otra desventaja que expresan, que es que las instrucciones en los cursos en línea no siempre son suficientemente claras, como lo indica esta misma maestra a continuación: 
En esos cursos que he entrado anteriormente sí me han dejado mucho que desear, o sea, ¿cómo te digo?, no te explican bien la actividad, o sea, tienes que, ahí sí es de que "amiga, ¿cómo es la actividad? o ¿cómo le hiciste?" cuando se supone que no debes tampoco tener esas dudas porque si presencialmente se nos hace difícil, ahora imagínate en línea, o sea, es mucho más difícil, entonces yo considero que tiene que ser un poquito más específico $(\mathrm{P} 2)$.

Como última desventaja mencionada está la ausencia de contacto directo entre las personas; una de las profesoras entrevistadas indica que un proceso de aprendizaje cara a cara es para ella más enriquecedor: "yo aprendo más cuando estoy platicando, cuando estoy conversando y cuando estoy interactuando, ya que cuando es en línea pues sí aprendo de igual manera, pero siento que es más enriquecedor cuando estás conviviendo con varias personas" (P4).

Es interesante el hecho de que el total de las informantes, a pesar de indicar que existen tanto ventajas como desventajas de la formación continua en línea, aun así, expresan preferir la opción presencial. Manifiestan que cuando menos les gustaría que, si es en línea, haya un componente presencial donde se les apoye con dudas y se les dé seguimiento cara a cara para fortalecer los procesos formativos. Así lo expresa una de las profesoras: "sí está bien la idea de hacerlo en línea porque hay maestros que no tienen acceso a ir a lo mejor a alguna parte, al centro, a tomar el curso, pero sí me gustaría eso, que a lo mejor fuera 50-50 o así presencial y subir proyectos" (P6).

\subsection{Requerimientos de participación de la formación continua en línea}

Los docentes reconocen que la modalidad en línea exige ciertos requerimientos para participar efectivamente; dos tipos fueron identificados: (1) materiales y (2) personales. Entre los primeros se enlistan: (a) el contar con una computadora o dispositivo electrónico desde el cual acceder a la red; y (b) una buena conectividad a internet. En cuanto a los requerimientos personales, se contemplan: (a) las habilidades digitales; (b) el compromiso e interés en la superación profesional; y (c) las habilidades para aprender autónomamente.

Respecto a las habilidades digitales la mayoría de las docentes consideran necesario contar con conocimientos básicos del funcionamiento de la computadora, de procesadores de textos y de estrategias de búsqueda de información en la red para participar en opciones formativas en línea; expresan que esto no les parece tan complicado puesto que generalmente estas iniciativas presentan tutoriales y apoyos que orientan en el manejo del entorno digital o plataforma. Explícitamente una de las maestras comenta: "no ocupas [sic] el saber tanto de una computadora, lo básico, cualquier persona puede acceder a ello y más ahorita [sic] que estamos en la era de la tecnología" (P2). A este mismo respecto, sobre las habilidades digitales, las entrevistadas más jóvenes expresaron tener facilidad para el manejo de la tecnología y buena actitud ante ella; por su parte, las docentes de mayor edad también mostraron disposición a su uso, como lo expresó, por ejemplo, una de las profesoras: “a mí me gusta, lo que no sé, lo preguntó, y me gusta buscarlo y tengo compañeros jóvenes, y [les digo] ¿qué pasa si le muevo aquí?, ¿si le muevo allá?” (P6).

Dentro de los requerimientos personales para participar en opciones formativas en línea, también mencionan el interés propio del docente y su compromiso por seguir aprendiendo y mejorando profesionalmente. Además, algunos informantes también hacen hincapié en que es necesario ser autónomos en el aprendizaje, para poder gestionar adecuadamente el tiempo y tener la disposición de cumplir con las actividades. Una de las profesoras lo expresa de la siguiente manera:

Tener el compromiso de iniciar el curso y de llevarlo a cabo como debería de ser [...] creo que es lo principal, porque a veces uno tiene 
el interés de querer, pero no es comprometido y ya de ahí ya no lleva uno correctamente la gestión de nuestro tiempo, le da prioridad a otras cosas. Entonces siempre tiene uno que tener muy en mente lo que se quiere (P4).

\subsection{Retos de la formación continua en línea de docentes}

El análisis de los datos permitió identificar cuatro retos que asociaron las docentes a la formación continua en línea: (1) el equipo tecnológico requerido; (2) temor al uso de la tecnología; (3) calidad de la oferta de formación en línea; (4) el valor o interés hacia la formación continua. El primer reto remite a la condición indispensable para acceder a este tipo de modalidad: la necesidad de contar con el equipamiento tecnológico requerido. Este incluye mínimamente materiales como una computadora y tener servicio de internet. El segundo reto tiene que ver con las habilidades tecnológicas necesarias; si bien, como se analizó en la categoría anterior de requerimientos, la mayoría de los profesores consideran que los conocimientos sobre las computadoras pueden ser mínimos, también reflexionan acerca de que muchos docentes aún manifiestan temor de incursionar en opciones de formación en línea; este temor, proviene precisamente de la creencia de que se carece de habilidades para manejar los dispositivos tecnológicos o de que no están tan acostumbrados a ellos, como lo expresan a continuación: "el principal reto es el de adoptar las tecnologías [...] hay maestros que no están tan familiarizados, porque están acostumbrados a que sea un curso presencial o un taller" (P4), "[...] hay muchos compañeros renuentes a perderle el miedo a la máquina (computadora), o sea, de ni siquiera iniciar el curso porque no saben; les da miedo la tecnología" (P6).

Un tercer reto identificado apunta hacia la generación de una oferta de formación continua en línea de calidad. Los docentes instan a las autoridades educativas a que les proporcionen iniciativas que cumplan con requisitos de calidad, que sean estrategias bien cimentadas, organizadas y que haya acompañamiento externo en el proceso. Consideran que esto sería un incentivo para continuar actualizándose a través de diferentes recursos. Así lo expresó una profesora:

El docente no le tiene miedo a la actualización, porque los conocimientos los tenemos, lo que pasa es que nos venden esas herramientas, nos exigen plataformas, nos exigen proyectos, nos exigen cosas, pero nosotros somos el piloto, nosotros somos el 'a ver si funciona' que aunado a la carga académica y administrativa nos dificultan más las cosas (P5).

Por último, el cuarto reto se relaciona con la revalorización misma de la formación continua. Las docentes entrevistadas expresaron que, desde su perspectiva, algunos de sus pares tienen un bajo nivel de participación general en las opciones formativas que hay disponibles; según como ellas lo expresan, esto puede relacionarse con el interés que cada quien manifiesta hacia su propia superación profesional, o bien con la eliminación de incentivos económicos que anteriormente estaban ligados a la formación continua pero que ya no están vigentes. En este sentido, se resalta la revalorización de la formación permanente como un tema importante a fin de fomentar la participación de los profesores en cualquier tipo de programa de formación permanente, sea presencial o en línea.

\subsection{Experiencias en la Colección de Aprendizajes Clave}

En esta categoría se recogen las impresiones de las informantes según lo que experimentaron en los MOOCs "Colección de Aprendizajes Clave", como parte de su formación para la implementación del Nuevo Modelo Educativo. A partir de los datos colectados, sus experiencias se incluyeron en cuatro subcategorías: (1) dimensión 
didáctico-pedagógica; (2) masividad y ausencia de tutor; (3) infraestructura tecnológica; y (4) el papel de la autoridad escolar (ver Figura 3 ), mismas que se describen a continuación.

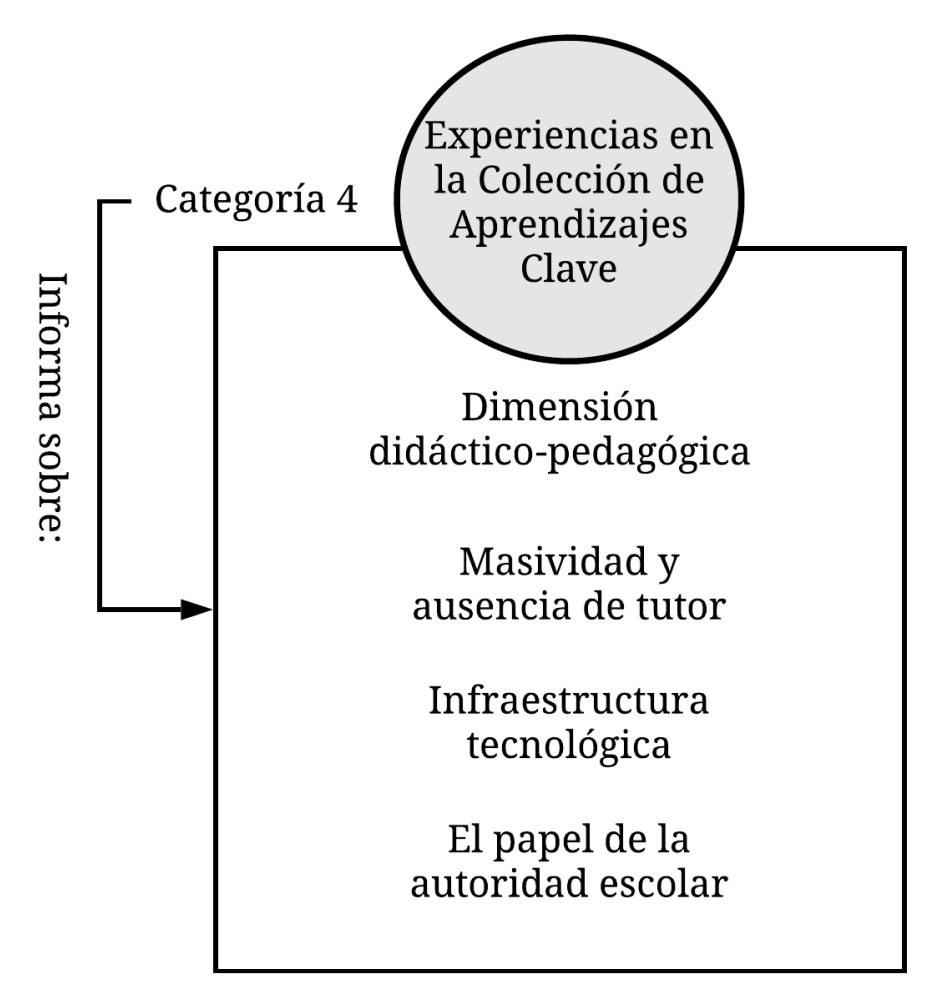

Figura 3. Experiencias en la Colección de Aprendizajes Clave

En cuanto a la dimensión didáctico-pedagógica, se recuperaron las experiencias de las cinco profesoras que participaron en los cursos en relación con los contenidos y actividades del curso. La mayoría de ellas coincidió en que el contenido era pertinente puesto que abordaba el Nuevo Modelo, lo que era una necesidad de actualización prioritaria en el momento en el que lo cursaron; pese a ello, según su opinión, este era de tipo más teórico que práctico. Señalaron que, aunque el contenido era extenso, el análisis se quedó en un nivel superficial; manifestaron que hubiera sido provechoso contar con más lecciones o bien con una segunda parte del curso. Por ejemplo, una de las profesoras expresó:

Sí logré aprender un poco, yo siento que faltó más del curso, como que darle seguimiento porque se quedó nada más en 10 lecciones y eso fue en vacaciones [...], pero el trabajo es diario, debió de haber habido segunda parte, no sé, haberlo manejado así: 'durante el ciclo va a haber otro curso y hay que subir evidencias y cosas así y proyectos', pero así como que quedó a medias, como un inicio, nada más se abordó así por encimita [sic] pero sí faltan muchos temas que abordar (P6).

Sobre las actividades tres profesoras señalaron algunas de estas como recursos útiles, mientras que a otras las describen como monótonas y cansadas; una de ellas expresó: "las lecturas muy tediosas la verdad, con letra pequeña, un poco largas" (P5). Además, indicaron que las orientaciones no siempre fueron precisas y adecuadas; por ejemplo, una de las maestras dijo: "yo tuve dudas sobre algunos temas y pues nadie me las pudo resolver" (P6), mientras que otra profesora indicó: "estaba un poco desorientada en ocasiones con algunos productos [...] a veces estaba enredoso [sic]..." (P4). Pese 
a estas observaciones, también se manifestó que las actividades favorecieron que los profesores compartieran puntos de vista sobre temas y experiencias.

Por ejemplo, la participación en foros fue una estrategia valorada ya que vieron en ellos precisamente la oportunidad de compartir ideas y experiencias con otros docentes. No obstante, consideraron que esto no se logró efectivamente, reconociendo que muchas aportaciones se hacían simplemente como requisito, sin existir una retroalimentación auténtica entre pares. Al respecto, una de las profesoras manifestó:

La plataforma tenía alrededor de tres foros que tenías que comentar a fuerzas [sic] para poder avanzar al siguiente módulo, tenías que terminar el módulo uno para avanzar al dos y así sucesivamente. Entonces cuando tú llegabas a ese foro supongamos: “¿tú qué piensas acerca de la educación?” y ya ponías tu expresión, tu idea y ya la mandabas y sí se supone que tenía que haber una retroalimentación entre nosotros para enriquecerla o para que [...] tuvieras más de dónde agarrar [sic] y realmente no, o sea, la subías y se iba llenando, se iba llenando, y eran miles de comentarios y ni siquiera te fijabas tú en los demás [...], lo que tú querías era subir y continuar, subir y continuar (P5).

Sobre la subcategoría de masividad y ausencia de tutor, se resalta que estos son dos características que distinguen particularmente a los MOOC; sobre estos elementos los profesores expresaron sus experiencias en los MOOCs cursados. Respecto a la masividad, se destacó que esta planteó algunos retos; por ejemplo, las profesoras sintieron que la tarea de organizarse con otros colegas para trabajar fue complicada debido a que no se conocían. En lo que corresponde a la ausencia de un tutor directo, en general las docentes se mostraron insatisfechas puesto que, desde su perspectiva, no hubo una figura que les brindara retroalimentación sobre su proceso de aprendizaje; expresaron que si bien sus actividades eran recibidas no se les proporcionaba una evaluación. Una de las profesoras comentó: "qué tiene que hubiera sido a la distancia, pero no hubo alguien que te dijera 'sabes qué, estás mal' o 'esto sí maestra, está muy bien, pero le faltó esto" (P6).

En cuanto a la subcategoría de infraestructura tecnológica, la mayoría de las docentes expresaron que la plataforma fue amigable y sencilla de utilizar; las actividades de cada módulo se desplegaban correctamente y se especificaba qué era lo que seguía por realizar. Sin embargo, también comentaron que experimentaron problemas técnicos en acciones como crear un usuario, cargar tareas, guardar cambios en las actividades, entre otras, lo que afectó negativamente su experiencia de aprendizaje. Sobre esto, una maestra comentó: "esa plataforma se saturaba constantemente, te sacaba, querías guardar y no te permitía, escribías y le dabas guardar y no se guardaba” (P5); mientras otra maestra dijo:

Se caía a cada ratito [sic] el sistema, no podías subir los trabajos [...] entraba en la madrugada y yo tenía mi trabajo en la computadora, pero lo quería subir y me levantaba a las cinco de la mañana, a las cuatro, y ya lo podía subir; a veces no (P6).

Estos problemas hicieron que el proceso de participación fuera desgastante. Comentaron que sentían estrés por realizar el trabajo y que los problemas con la plataforma lo incrementaban.

Por último, en cuanto a la categoría sobre el papel de la autoridad escolar, las participantes manifestaron que durante la implementación del programa la comunicación de las autoridades educativas inmediatas fue escasa. Se les hizo saber que debían tomar el curso, pero no les dieron mayores informes del proceso. Así lo expresó una profesora: 
La información bajó directamente de nuestro director, él nos lo hizo saber, nomás [sic] nos dijo que era un curso, que entráramos a la página, que lo teníamos que hacer, más sin embargo no se nos dieron los requisitos, lineamientos, ni nada, simplemente se nos mencionó que teníamos que realizarlo; ya el maestro tenía que tener la iniciativa de investigar un poco más para irse empapando [sic] de ese tema (P5).

Sobre esto mismo, algunas profesoras indicaron que primeramente se les hizo una invitación libre, pero después se les indicó que era un requisito. Otras maestras, por su parte, comentaron que con ellos sucedió a la inversa, es decir, primero se les presentó como una participación obligatoria y luego se les dijo que era opcional. Este tipo de comunicación provocó incertidumbre entre las profesoras; y ante estas dificultades y dudas en el proceso, las maestras señalaron que el apoyo por parte de las autoridades educativas para solventarlas fue escaso, indicándoles sencillamente que cualquier inconveniente debían tratarlo a través de las mesas de ayuda en la plataforma. Esto hace notar que las experiencias en los MOOCs fueron diversas para las profesoras entrevistadas; tuvieron situaciones que dificultaron su participación, pero también variados aspectos favorables para su aprendizaje.

\section{DISCUSIÓN}

Fishman (2016) sostiene que el éxito o la calidad de un programa de desarrollo profesional que involucre tecnología, no depende únicamente de la tecnología empleada, sino que deben tenerse en cuenta aspectos como los participantes y su entorno. En este estudio, el reconocimiento de una actitud de apertura hacia la formación en línea por parte de las profesoras fue uno de los hallazgos importantes. Los principales aspectos positivos que las profesoras ven en la modalidad en línea son la flexibilidad de tiempo y espacio, y la reducción de costos. Esto coincide con los hallazgos obtenidos por otras investigaciones (Gómez y Silas, 2016; Ramírez, Casillas, y Aguirre, 2018). Esta apertura es fundamental ya que las nuevas propuestas de desarrollo profesional docente conciben al profesor como un aprendiz que está en el centro de los procesos formativos; y estos nuevos ambientes de aprendizaje, de acuerdo con Tancredi (2009), conforman un binomio indisoluble con las TIC. Así, la visión optimista hacia la adopción de la formación en línea se reconoce como necesaria para acometer los desafíos de la Sociedad del Conocimiento, en la cual los docentes tienen la tarea de aprender y enseñar con las TIC.

No obstante, a pesar de la postura abierta ante la formación en línea, otro hallazgo importante de este estudio fue que los docentes siguen prefiriendo el esquema presencial de formación. Las profesoras ven en la modalidad en línea ventajas de gran valor, sin embargo, esto no necesariamente se traduce en la preferencia de esta alternativa frente a la modalidad presencial. Se percibe a la formación en línea como una manera de apoyar, más no de sustituir, a la formación presencial. Al respecto, Calatayud (2007) destaca que tres de las razones por las que el docente no se forma a través del e-learning son (1) el miedo a lo desconocido, debido a la poca familiarización con esta modalidad; (2) la creencia de que este tipo de modalidad no presenta el mismo reconocimiento que la formación presencial; y (3) la carencia de conocimientos y habilidades informáticas de los docentes para poder formarse a través de la tecnología. Lo anterior plantea retos principalmente relacionados con brindar apoyo a los docentes para emigrar de esquemas tradicionales de aprendizaje hacia nuevos espacios colaborativos a través de las herramientas que ofrece la educación en línea.

Concretamente, de la experiencia en la colección de MOOCs de Aprendizajes Clave, los docentes resaltan la masividad y la ausencia de un tutor como dos aspectos cruciales que delinearon su participación. Las profesoras enfrentaron algunas dificultades ante 
estas dos características básicas de los MOOCs; por una parte, la masividad generó inconvenientes al tratar de organizarse en grupos debido al desconocimiento personal de sus compañeros de curso, resultados similares obtuvieron Salmon et al. (2015) quienes agregan que esto puede deberse a que la gran cantidad participantes puede provocar una interacción impersonal. En tanto, la ausencia de un tutor generó insatisfacción al no contar con alguien que les brindara retroalimentación sobre su proceso de aprendizaje. Sobre este aspecto, Chiappe-Laverde et al. (2015) mencionan que la ausencia de un tutor en los MOOCs puede solucionarse al utilizar diferentes estrategias como la retroalimentación entre pares. En ambos casos estos hallazgos remiten a la importancia que tiene el diseño instruccional para atender ambas características, de tal manera que los MOOCs fomenten la colaboración y permitan la evaluación y retroalimentación eficiente entre participantes.

Los hallazgos anteriores también ponen de relieve la importancia de considerar el tipo de formación que han recibido los docentes en México, más cercana a los estilos de enseñanza y aprendizaje tradicionales. Este estilo generalmente está alejado de las propuestas de aprendizaje de los MOOCs, las cuales de acuerdo con Kop (2011) demandan contar con habilidades digitales diferentes a la mera búsqueda y validación de información, como el ser capaz de editar y producir información, y el comunicarse y colaborar con otros de nuevas formas. Asimismo, la literatura sobre las experiencias de los aprendices muestra que el contar con un nivel avanzado de habilidades digitales es uno de los aspectos más importantes que definen la experiencia del participante en un MOOC (Kop y Fournier, 2010). Esto implica que los MOOCs agregan requerimientos a los que habitualmente exige la propia formación en línea lo cual constituye un desafío para su utilización en la formación continua de profesores.

Finalmente, se destaca la importancia del papel de la autoridad educativa; la escasa orientación y participación de la autoridad educativa influyó de manera poco favorable en la experiencia de las profesoras en la "Colección de Aprendizajes Clave". Para atender necesidades nacionales de formación por medio de estrategias a gran escala, como es el caso de los MOOCs, se ha evidenciado que la orientación de la autoridad es un factor clave para el éxito de este tipo de estrategias (Magaña-Valladares, González-Robledo, et al., 2018; Magaña-Valladares, Rosas-Magallanes, et al., 2018). Lo anterior pone de manifiesto la relevancia de un compromiso conjunto, que permita la conformación de un equipo entre autoridades educativas, diseñadores instruccionales, facilitadores y líderes, que asegure la calidad de la implementación de estrategias de esta naturaleza para los profesores.

\section{CONCLUSIONES}

La formación en línea se ha convertido en una opción educativa de interés. Ante la preocupación creciente por abordar los desafíos de la profesión docente, se le ha utilizado para brindar oportunidades de formación continua y desarrollo profesional a este importante sector de la sociedad. Como ya se ha argumentado, el éxito o la calidad de un programa de desarrollo profesional que involucre tecnología, no depende sólo de los recursos tecnológicos sino también de las características de los participantes y de su entorno. Por ello, este estudio exploró la percepción y experiencias de los profesores en su formación continua en línea.

Se reconoce que nuevos estudios deberán realizarse para comprender, desde otras aproximaciones metodológicas y de acuerdo con otras figuras educativas, los aspectos que contribuyen a una formación en línea de calidad para los profesores. Dado que este estudio significó para los investigadores un primer acercamiento al contexto, su principal reto, y la vez una limitante de esta investigación, fue el acceso a una mayor cantidad de participantes que pudieran informar sobre su experiencia en la formación en línea, particularmente en los MOOCs. 
No obstante, a partir de los hallazgos identificados, es posible compartir algunas reflexiones. Las perspectivas del profesorado hacia las estrategias de formación en línea se ven influenciadas por sus habilidades digitales y, particularmente, por las experiencias previas que tienen en este tipo de estrategias; aunque los profesores reconozcan ventajas de la formación en línea, experiencias poco exitosas en este formato influyen directamente en su disposición y deseo por participar en ellas. En concreto, sobre el uso de MOOCs para la formación docente, se destacan el acompañamiento externo y el diseño instruccional como dos aspectos fundamentales para el éxito de este tipo de cursos.

La actualización docente a través de medios virtuales en México tiene poco tiempo de haber surgido. Si se desea apostar por la formación continua en línea, es necesario apoyar a los docentes con estrategias que paulatinamente les permitan transitar de un esquema presencial a uno virtual. Esto será posible en la medida en que se conjuguen los recursos y apoyo de parte de las instancias de formación docente, con el compromiso y apertura de los propios docentes hacia nuevas alternativas de favorecimiento de su desarrollo profesional. En este sentido, en futuras investigaciones se propone continuar profundizando en la comprensión de las condiciones que benefician la implementación de la formación continua en línea en los docentes, concretamente a través de los MOOCs, mismos que se estima continuarán siendo una tendencia educativa.

\section{REFERENCIAS}

Alemán, L. Y., Sancho-Vinuesa, T., y Gómez, M. G. (2015). Indicadores de calidad pedagógica para el diseño de un curso en línea masivo y abierto de actualización docente. RUSC. Universities and Knowledge Society fournal, 12(1), 104-119.

http://dx.doi.org/10.7238/rusc.v12i1.2260

Álvarez Gayou, J. L. (2003). Cómo hacer investigación cualitativa. Fundamentos y metodología. México: Paidós.

Ávalos, B. (2007). El desarrollo profesional continuo de los docentes: lo que nos dice la experiencia internacional y de la región latinoamericana. Pensamiento educativo. Revista de Investigacion Educacional Latinoamericana, 41(2), 77-99. Descargado de https://pensamientoeducativo.uc.cl/index.php/pel/article/view/417

Avello-Martínez, R., y Duart, J. M. (2016). Nuevas tendencias de aprendizaje colaborativo en e-learning. Claves para su implementación efectiva. Estudios pedagógicos, 42(1), 271-282. http://dx.doi.org/10.4067/S0718-07052016000100017

Benet, A., Sanahuja, A., García, I., y Nieto, R. (2018). Nuevos horizontes formativos: una experiencia del MOOC como recurso en la formación continua. Apertura, 10(1), 88-103. http://dx.doi.org/10.18381/ap.v10n1.1151

Cabero-Almenara, J., Gallego-Pérez, O., Puentes, A., y Jiménez, T. (2018). La “Aceptación de la Tecnología de la Formación Virtual” y su relación con la capacitación docente en docencia virtual. EDMETIC. Revista de Educación Mediática y TIC, 7(1), 225-241. https://doi.org/10.21071/edmetic.v7i1.10028

Calatayud, M. A. (2007, 24 de 09). Apostar por la formación e-learning: entre la utopía y la realidad. [Blog post]. Descargado de https://www.educaweb.com/noticia/2007/09/24/ apostar-formacion-learning-utopia-realidad-2523/

Castaño, C., y Cabero, J. (2013). Enseñar y aprender en entornos m-learning. En and others (Ed.), . Madrid: Síntesis.

Chiappe-Laverde, A., Hine, N., y Martínez-Silva, J. A. (2015). Literatura y práctica: una revisión crítica acerca de los MOOC. Comunicar. Revista Científica de Comunicación y Educación(44), 9-18. https://doi.org/10.3916/C44-2015-01

Chofré, L. A., y Calvet, R. (2014). Una experiencia práctica de la utilización de MOOC como una herramienta educativa y de formación continua del profesorado. En XI fornadas Internacionales de Innovación Universitaria (pp. 330-337). 
Colomer-Rubio, J. C. ., Sáiz-Serrano, J., y Bel-Martínez, J. (2018). Competencia digital en futuros docentes de Ciencias Sociales en Educación Primaria: análisis desde el modelo TPACK. Educatio Siglo XXI, 36, 107-128. https://doi.org/10.6018/j/324191

Cordero-Arroyo, G. (2015). La formación continua y la evaluación del desempeño docente en el marco de la Reforma Educativa mexicana. , 32, 5-10. Descargado de http://www.cucs.udg.mx/revistas/edu_desarrollo/anteriores/32/32_Cordero.pdf

Creswell, J. W. (2007). Qualitative inquiry research design. Choosing among five approaches. California (EEUU): SAGE Publications.

Davini, M. (2015). La formación en la práctica docente. Buenos Aires: Paidós.

Dede, C. (2006). Online Teacher Professional Development. Cambridge: Harvard Education Press.

Dede, C., Ketelhut, D., Whitehouse, P., Breit, L., y McCloskey, E. (2009). A research agenda for online teacher professional development. Journal of Teacher Education, 60, 8-19. https://doi.org/10.1177/0022487108327554

DGFC. (2018). Estrategia Estatal de Formación Continua. Descargado de https://dgfc.basica.sep.gob.mx/multimedia/RSC/BASICA/Documento/201810/ 201810-RSC-BUTGN9I7na-SONORA_ESTRATEGIA2018_FEB.pdf

DOF. (2017). Acuerdo 12/10/17 por el que se establecen el Plan y los Programas de estudio para la Educación Básica: Aprendizajes Clave para la Educación Integral. Descargado de https://dof.gob.mx/nota_detalle.php?codigo=5578281\&fecha=08/11/2019

Fishman, B. (2016). Possible Futures for Online Teacher Professional Development. En C. Dede, A. Eisenkraft, K. Frumin, y A. Hartley (Eds.), Teacher Learning in the Digital Age: Online Professional Development in STEM Education (pp. 13-30). Cambridge: Harvard Education Press.

Forman, J., y Damschroder, L. (2008). Qualitative content analysis. En L. Jacoby y L. A. Siminoff (Eds.), Empirical Methods for Bioethics: A Primer (pp. 39-62). Amsterdam: Elsevier.

Gárate-Carrillo, M., y Cordero-Arroyo, G. (2019). Apuntes para caracterizar la formación continua en línea de docentes. REXE. Revista de Estudios y Experiencias en Educación, 18(36), 2019-221. https://doi.org/10.21703/rexe.20191836garate10

García, P. J., Ibarra, C., y Sandoval, K. M. (2018). Cursos MOOC: Una alternativa de formación docente en CONALEP. En E. R. M. del Collado . (Ed.), Cursos masivos abiertos en línea MOOCs: el caso de México (pp. 169-182). Editorial Brujas.

Gómez, L. F., y Silas, J. C. (2016). La comunidad virtual de práctica. Alternativa para la formación continua de profesores. CPU-e. Revista de Investigación Educativa, 22, 28-51. Descargado de http://www.scielo.org.mx/pdf/cpue/n22/1870-5308-cpue-22-00028.pdf CPU-e. Revista de Investigación Educativa.

Gonçalves, V., Chumbo, I., Torres, E., y Gonçalves, B. (2016). Teacher education through MOOC: a case study. 9th annual International Conference of Education, Research and Innovation. ICERI2016 Proceedings (pp. 8350-8358). IATED.

https://doi.org/10.21125/iceri.2016

Imbernón, F. (2001). La profesión docente ante los desafíos del presente y del futuro. En C. Marcelo (Ed.), La función docente (pp. 27-45). Madrid: Síntesis Educación. Madrid: Síntesis Educación.

Jansen, D., Schuwer, R., Teixeira, A., y Aydin, C. H. (2015). Comparing MOOC adoption strategies in Europe: Results from the HOME project survey. International Review of Research in Open and Distributed Learning, 16(6), 116-136.

https://doi.org/10.19173/irrodl.v16i6.2154

Ji, Z., y Cao, Y. (2016). A Prospective study on the application of MOOC in teacher professional development in China. Universal fournal of Educational Research, 4(9), 2061-2067.

Jobe, W. O., Stlund, C., y Svensson, L. (2014). MOOCs for professional teacher development. Society for Information Technology \& Teacher Education International Conference (pp. 
1580-1586).

Kannan, K., y Narayanan, K. (2015). A structural equation modelling approach for massive blended synchronous teacher training. Journal of Educational Technology \& Society, 18(3), 1-15.

Karlsson, N., Godhe, A., Bradley, L., y Lindström, B. (2014). Professional development of teachers in a MOOC. En C. Liu et al. (Eds.), Proceedings of the 22nd International Conference on Computers in Education (pp. 868-877). Descargado de https://pellepedagog.se/wp-content/uploads/2015/01/ Professional-Development-of-Teachers-in-a-MOOC.pdf

Kop, R. (2011). The Challenges to Connectivist Learning on Open Online Networks: Learning Experiences during a Massive Open Online Course. International Review of Research in Open and Distributed Learning, 12(3), 19-38. https://doi.org/10.19173/irrodl.v12i3.882

Kop, R., y Fournier, H. (2010). New Dimensions to Self-directed Learning in an Open Networked Learning Environment. International fournal of Self-Directed Learning, 7(2), 1-20. Descargado de https://nrc-publications.canada.ca/eng/view/object/ ?id=c4dc46c9-ef59-46b8-af01-4a7fec44b023

Koukis, N., y Jimoyiannis, A. (2019). MOOCS for teacher professional development: exploring teachers' perceptions and achievements. Interactive Technology and Smart Education, 16(1), 74-91. https://doi.org/10.1108/ITSE-10-2018-0081

Koutsodimou, K., y Jimoyiannis, A. (2015). MOOCs for teacher professional development: investigating views and perceptions of the participants. 8th International Conference of Education, Research and Innovation. ICERI2015 Proceedings (pp. 6968-6977). IATED.

Langseth, I., y Haugsbakken, H. (2016). Introducing blended learning MOOC-a study of one bMOOC in Norwegian teacher education. En T. Brinda, N. Mavengere, I. Haukijärvi, C. Lewin, y D. Passey (Eds.), Stakeholders and Information Technology in Education. SaITE 2016. IFIP Advances in Information and Communication Technology (Vol. 493, pp. 59-71). Cham: Springer. Portugal. https://doi.org/10.1007/978-3-319-54687-2_6

Laurillard, D. (2016). The educational problem that MOOCs could solve: professional development for teachers of disadvantaged students. Research in Learning Technology, 24(1), 1-24. https://doi.org/10.3402/rlt.v24.29369

Lugo, M., Lion, C., y Vaillant, D. (2018). Desafíos y tendencias en las políticas digitales para el desarrollo profesional de docentes. Descargado de https://eventos.iiep.unesco.org/comment/2416 Archivo de video.

Magaña-Valladares, L., González-Robledo, M. C., Rosas-Magallanes, C., Mejía-Arias, M. A., Arreola-Ornelas, H., y Knaul, F. (2018). Training primary health professionals in breast cancer prevention: evidence and experience from Mexico. Fournal of Cancer Education, 33(1), 160-166. https://doi.org/10.1007/s13187-016-1065-7

Magaña-Valladares, L., Rosas-Magallanes, C., Montoya-Rodríguez, A., Calvillo-Jacobo, G., Alpuche-Arande, C. M., y García-Saisó, S. (2018). A MOOC as an immediate strategy to train health personnel in the cholera outbreak in Mexico. BMC Medical Education(111), 18-18. https://doi.org/10.1186/s12909-018-1215-1

Marauri, P. M. (2014). La figura de los facilitadores en los Cursos Online Masivos y Abiertos (COMA /MOOC): nuevo rol profesional para los entornos educativos en abierto. Revista Iberoamericana de Educación a Distancia, 17(1), 35-67.

https://doi.org/10.5944/ried.17.1.11573

Marín Trejo, R. (2017). Diseño y validación de un instrumento de evaluación de la competencia digital docente (Tesis Doctoral, Universitat de les Illes Balears, Islas Baleares (España)). Descargado de http://hdl.handle.net/11201/148469

Misra, P. K. (2018). MOOCs for teacher professional development: Reflections and suggested actions. Open Praxis, 10(1), 67-77. http://dx.doi.org/10.5944/openpraxis.10.1.780

Navareño, P., y Rincón, J. (2017). Contribuciones de la plataforma online TeachersPro al desarrollo profesional del entorno personal de aprendizaje (E. J. R.-P. . y J. S.-R. y E. Sánchez-Rivas ., Eds.). Innovación docente y uso de las TIC en educación. Málaga: 
UMA Editorial.

NRC. (2007). Enhancing Professional Development for Teachers: Potential Uses of Information Technology: Report of a Workshop. https://doi.org/10.17226/11995

OCDE. (2019). TALIS 2018. Estudio internacional de la enseñanza y el aprendizaje. Informe español. Descargado de https://www.educacionyfp.gob.es/inee/dam/jcr: 047dfc41-acf6-444a-8e4e-4e5916343a88/talis2018-online-20190807.pdf

Palmer, M. (2015, 16 de 03). Are we missing opportunities to engage teachers with MOOCs? [Blog post]. Descargado de https://blog.edx.org/are-we-missing-opportunities-engage/

Patru, M., y Balaji, V. (2016). Making Sense of MOOCs: A guide for policy-makers in developing countries. Commonwealth of Learning / UNESCO. Descargado de https://unesdoc.unesco.org/ark:/48223/pf0000245122

Ramírez, A., y Casillas, M. (2018). Diseño de un MOOC de habilitación tecnológica de docentes. En E. R. M. del Collado (Coord . (Ed.), Cursos masivos abiertos en línea MOOCs: el caso de México (pp. 109-122). Editorial Brujas.

Ramírez, A., Casillas, M. A., y Aguirre, I. R. (2018). Habilitación tecnológica de profesores universitarios y docentes de educación básica. Apertura, 10(2), 124-139. Descargado de http://www.scielo.org.mx/pdf/apertura/v10n2/2007-1094-apertura-10-02-124.pdf

Rivera-Vázquez, N., y Ramírez-Montoya, M. S. (2015).

Salmon, G., Gregory, J., Lokuge, K., y Ross, B. (2015). Experiential online development for educators: The example of the Carpe Diem MOOC. British fournal of Educational Technology, 46(3). https://doi.org/10.1111/bjet.12256

Samperio, V., y Barragán, J. (2018). Análisis de la percepción de docentes, usuarios de una plataforma educativa a través de los modelos TPACK, SAMR y TAM3 en una institución de educación superior. Apertura, 10(1), 116-131. https://doi.org/10.1111/bjet.12256

Sandín, M. P. (2003). Investigación Cualitativa en Educación. Fundamentos y tradiciones. España: McGraw-Hill.

Selwyn, N., Bulfin, S., y Pangrazio, L. (2015). Massive open online change? Exploring the discursive construction of the 'MOOC' in newspapers. Higher Education Quarterly, 69(2), 175-192. https://doi.org/10.1111/hequ.12061

SEP. (2018). Estrategia Nacional de Formación Continua 2018. México.

Tancredi, B. (2009). Nuevos ambientes de aprendizaje para el desarrollo profesional docente. En C. V. de Medrano y D. E. Vaillant (Eds.), Aprendizaje y desarrollo profesional docente (pp. 159-170). OEI. Descargado de http://repositorio.minedu.gob.pe/handle/123456789/4622

UNESCO. (2015). Estrategia de Educación de la UNESCO 2014-2021. Descargado de https://unesdoc.unesco.org/ark:/48223/pf0000231288_spa

Vicenteño, D. (2018, 16 de 01). Nuevo Modelo Educativo, con avance del 95 por ciento: SEP. Descargado de https://www.excelsior.com.mx/nacional/2018/01/16/1214082

Wang, Q., Chen, B., Fan, Y., y Zhang, G. (2018). MOOCs as an alternative for teacher professional development: Examining learner persistence in one Chinese MOOC. Beijing: Peking University. Descargado de http://dl4d.org/wp-content/uploads/2018/05/China-MOOC.pdf 